\title{
Steady-state phase diagram of a weakly driven chiral-coupled atomic chain
}

\author{
H. H. Jen $\odot^{*}$ \\ Institute of Physics, Academia Sinica, Taipei 11529, Taiwan
}

(Received 28 September 2019; published 29 January 2020)

\begin{abstract}
A chiral-coupled atomic chain of two-level quantum emitters allows strong resonant dipole-dipole interactions, which enables significant collective couplings between every other emitter. We numerically obtain the steady-state phase diagram of such a system under weak excitations, where interaction-driven states of crystalline orders, edge or hole excitations, and a dichotomy of chiral flow are identified. We distinguish these phases and regions by participation ratios and structure factors, and find two critical points which relate to decoherence-free subradiant sectors of the system. We further investigate the transport of excitations and the emergence of crystalline orders under spatially varying excitation detunings, and present nonergodic butterflylike system dynamics in the phase of extended hole excitations with a signature of persistent subharmonic oscillations. Our results demonstrate the interaction-induced quantum phases of matter with chiral couplings, and pave the way toward simulations of many-body states in nonreciprocal quantum optical systems.
\end{abstract}

DOI: 10.1103/PhysRevResearch.2.013097

\section{INTRODUCTION}

A chiral-coupled atomic system [1,2] from an atom-fiber [3] or an atom-waveguide [4,5] interface presents the capability to engineer the directionality of light transmissions. This leads to a broken time-reversal symmetry of light-matter couplings, and results in nonreciprocal decay channels. Unidirectional coupling [6] can therefore be enabled by spin-momentum locking [7,8], where light propagation highly correlates to its transverse spin angular momentum. Such a one-dimensional (1D) nanophotonics system has been studied to create mesoscopic quantum correlations [9] and quantum spin dimers [10-12], to enable simulations of long-range quantum magnetism [13], and to manifest emerging universal dynamics [14] and strong photon-photon correlations [15]. These compelling predictions rely on the emergence of infinite-range resonant dipole-dipole interactions (RDDIs) [16] in 1D system-reservoir interactions, in huge contrast to the ones in free space, which decrease with an interatomic distance in the long range [17].

The nonreciprocal decay channels of chiral-coupled systems can be tuned by external magnetic fields [3-5], such that the amount of light transmissions in the allowed direction can be controlled by the internal states of quantum emitters [3]. This can be attributed to reservoir engineering, which has spurred many interesting studies of nonequilibrium phase transitions in driven-dissipative quantum systems [18-20]. In such open systems, a self-organized supersolid phase [21]

\footnotetext{
*sappyjen@gmail.com

Published by the American Physical Society under the terms of the Creative Commons Attribution 4.0 International license. Further distribution of this work must maintain attribution to the author(s) and the published article's title, journal citation, and DOI.
}

of a Bose-Einstein condensate can be realized by coupling to an optical cavity, and exotic spin phases and multipartite entangled states respectively can be dissipatively prepared in laser-excited Rydberg atoms [22] and ions [23]. Under engineered dissipations, Majorana edge modes as topological states of matter $[24,25]$ and critical phenomena at steady-state phase transitions [26] are also predicted in lattice fermions. These quantum phases of matter under nonequilibrium phase transitions show the potential to explore dynamical phases driven by competing dissipation and interaction strengths [27].

Here, we consider two-level quantum emitters coupled to a nanofiber or waveguide with equal interatomic distances. We obtain the steady-state phase diagram of such a chiral-coupled atomic chain in the low saturation limit, determined by two competing parameters of directionality and the relative strength of the dissipative and coherent parts of dipole-dipole interactions. The interaction-driven phases include states with extended distributions (ETDs), crystalline orders (COs), bi-edge/hole excitations (BEEs/BHEs), and a region of chiral-flow dichotomy (CFD), which we classify by participation ratios and structure factors. Two critical points are also located, where the time to reach steady states is longer than the power-law dependence of the system sizes. This critically slow equilibrium of system dynamics relates to the decoherence-free sectors of the eigenspectrum. We further explore the possibility to relocate the atomic excitations via spatially varying field detunings or excitation directions. Finally, in the phases of BEE and BHE, nonergodic signatures of subharmonic oscillations emerge, and we specifically present a butterflylike system dynamics as an example. The steadystate phases and their dynamical evolutions investigated here present a distinct interplay between RDDIs and directionality of a chiral-coupled atomic chain, which give insights into the preparations and simulations of many-body states in nonreciprocal quantum optical systems. 

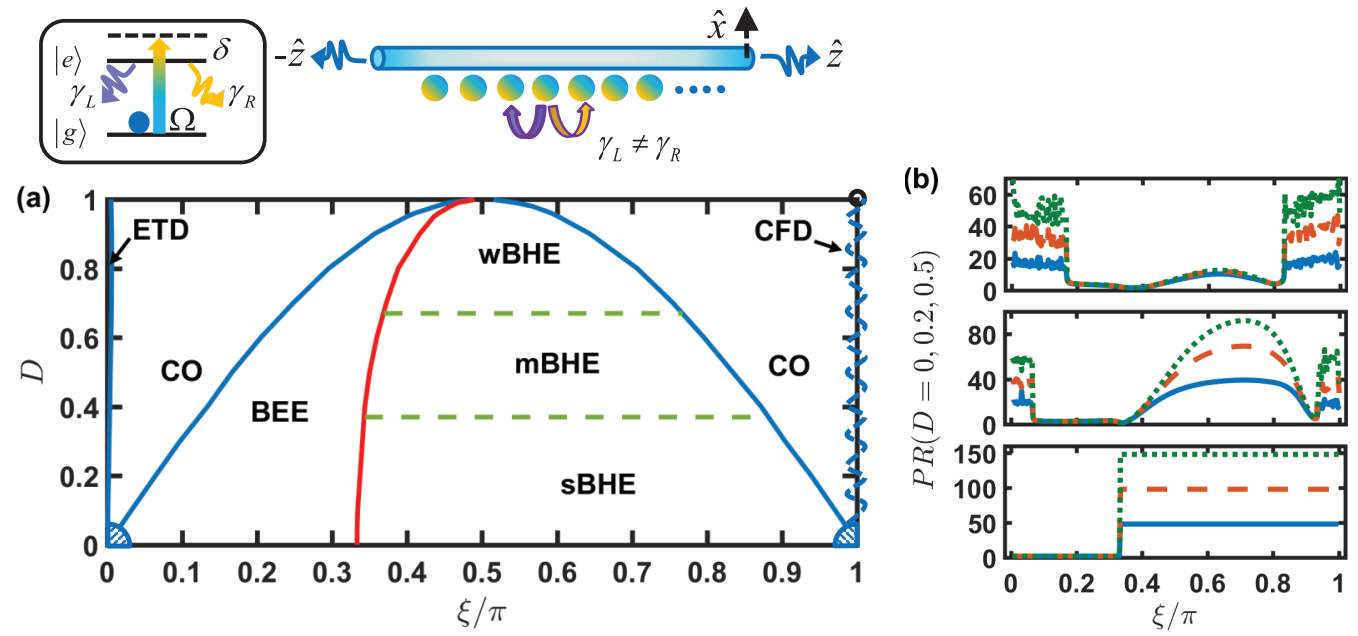

FIG. 1. Steady-state phase diagram of a weakly driven chiral-coupled atomic chain at $\theta_{s}=\pi / 2$ and $\delta_{\mu}=0$. The chiral-coupled system consists of two-level quantum emitters with nonreciprocal decay rates of $\gamma_{L} \neq \gamma_{R}$, mediated by a nanofiber or waveguide. (a) In the steady state, the phases of extended distributions (ETDs), finite crystalline orders (COs), bi-edge/hole excitations (BEEs/BHEs), and a region of chiral-flow dichotomy (CFD, wavy dashes) are identified under parameter spaces of directionality factor $D$ and dimensionless interatomic distance $\xi$. For different scaling parameters $\alpha$ of participation ratios on the number of atoms $\left(N^{\alpha}\right)$, we further separate the BHE phase into strong (s), moderate (m), and weak (w) regimes with $\alpha>0.5$ (below $D \approx 0.37$ ), $0.1<\alpha<0.5$, and $\alpha<0.1$ (above $D \approx 0.67$ ), respectively. The shaded regions at the two lower corners of the diagram represent the critical regimes. (b) Participation ratios (PRs) at different horizontal cuts of the diagram (increasing $D$ from the lower to upper panels), showing abrupt changes crossing the CO phases, with $N=50$ (solid line), 100 (dashes), and 150 (dots).

\section{THEORETICAL MODEL}

We consider a generic driven-dissipative model in Lindblad forms for a 1D chiral-coupled atomic chain [12] in Fig. 1,

$$
\frac{d \rho}{d t}=-\frac{i}{\hbar}\left[H_{S}+H_{L}+H_{R}, \rho\right]+\mathcal{L}_{L}[\rho]+\mathcal{L}_{R}[\rho] .
$$

The external and coherent field excitation is denoted as

$$
H_{S}=\hbar \sum_{\mu=1}^{N} \Omega e^{i k_{s} \cos \theta_{s} r_{\mu}}\left(\sigma_{\mu}+\sigma_{\mu}^{\dagger}\right)-\hbar \sum_{\mu=1}^{N} \delta_{\mu} \sigma_{\mu}^{\dagger} \sigma_{\mu},
$$

which drives $N$ two-level quantum emitters $(|g\rangle$ and $|e\rangle$ being the ground and excited state, respectively) with a Rabi frequency $\Omega$ and spatially dependent detunings $\delta_{\mu}$, and the dipole operators are $\sigma_{\mu}^{\dagger} \equiv|e\rangle_{\mu}\langle g|$ with $\sigma_{\mu}=\left(\sigma_{\mu}^{\dagger}\right)^{\dagger} . k_{s}$ denotes the wave vector, and a uniform excitation angle $\theta_{s}$ quantifies the traveling phases of the driving field from side excitations on the $\hat{y}-\hat{z}$ plane. The coherent parts of the RDDI in Eq. (1) are

$$
H_{L(R)}=-i \hbar \frac{\gamma_{L(R)}}{2} \sum_{\mu<(>) v}^{N}\left(e^{i k_{s}\left|r_{\mu}-r_{\nu}\right|} \sigma_{\mu}^{\dagger} \sigma_{\nu}-\text { H.c. }\right) \text {, }
$$

and the dissipative ones in the Lindblad forms are

$$
\begin{aligned}
\mathcal{L}_{L(R)}[\rho]= & -\frac{\gamma_{L(R)}}{2} \sum_{\mu, v}^{N} e^{\mp i k_{s}\left(r_{\mu}-r_{v}\right)} \\
& \times\left(\sigma_{\mu}^{\dagger} \sigma_{\nu} \rho+\rho \sigma_{\mu}^{\dagger} \sigma_{\nu}-2 \sigma_{\nu} \rho \sigma_{\mu}^{\dagger}\right) .
\end{aligned}
$$

They determine the collective energy shifts and decay rates, respectively, which mediate the whole system in an infinite range of sinusoidal forms, and the subscripts $L(R)$ label the left-(right)-propagating decay channels.
We proceed to solve for the steady-state solutions of Eq. (1) in the low saturation limit, which truncates the hierarchycoupled equations to self-consistently coupled dipole operators. In the Appendix, we show the details of the hierarchy-coupled equations, which are typical in the treatment of quantum electrodynamics and involve moments of system observables up to $N$ th order. They arise, for example, in dissipative quantum optical $[28,29]$ or condensed matter systems [30], whenever higher-order radiative couplings are considered. We then obtain steady-state $\vec{\sigma}^{(s)}$ satisfying $\dot{\sigma}_{\mu}=0$,

$$
\vec{\sigma}^{(s)}=i \Omega M^{-1} e^{i k_{s} \cos \theta_{s} \vec{r}},
$$

where $\vec{r}$ denotes the atomic distributions. In the coupling matrix $M$, the asymmetry of off-diagonal matrix elements arises due to unequal $\gamma_{L(R)}$, and they are

$$
M_{\mu, v}= \begin{cases}-\gamma_{L} e^{i k_{s}\left|r_{\mu, v}\right|}, & \mu<v, \\ i \delta_{\mu}-\frac{\gamma_{L}+\gamma_{R}}{2}, & \mu=v, \\ -\gamma_{R} e^{i k_{s}\left|r_{\mu, v}\right|}, & \mu>v,\end{cases}
$$

where $r_{\mu, v}=r_{\mu}-r_{v}$. From Eq. (6), we determine the interaction-driven quantum phases of matter which mainly rely on the interplay between the chirality of chiral-coupled systems and RDDI determined by interatomic distances. The external spatially varying excitation detunings and excitation angles play extra roles in relocating the atomic excitations and imprinting extra phases on the atoms, which we investigate below.

\section{STEADY-STATE PHASE DIAGRAM}

We use the directionality factor $D \equiv\left(\gamma_{R}-\gamma_{L}\right) / \gamma$ [3] to quantify the degree of light transmissions of the system with 


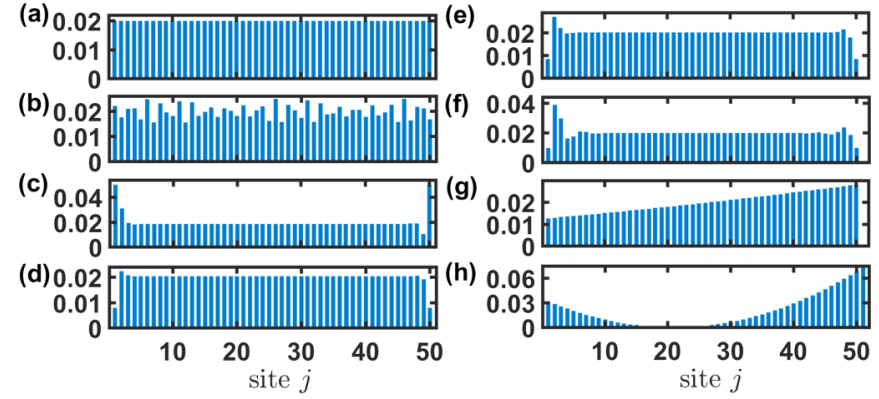

FIG. 2. Excited-state distributions of various phases in Fig. 1. We show some examples of steady-state distributions in the phases of (a) ETD at $D=0.2, \xi / \pi=0$, (b) CO at $D=0.2, \xi / \pi=0.02$, (c) BEE at $D=0.2, \xi / \pi=0.2$, (d) strong BHE (sBHE) at $D=0.2$, $\xi / \pi=0.6$, (e) moderate BHE (mBHE) at $D=0.5, \xi / \pi=0.6$, (f) weak BHE (wBHE) at $D=0.8, \xi / \pi=0.6$, and in a region of CFD at $D=0.2, \xi / \pi=1$ for (g) $N=50$ and (h) $N=51$. In all plots $N=50$ except in (h).

a normalized decay channel $\gamma_{R}+\gamma_{L}=\gamma . D= \pm 1$ and 0 present the unidirectional $[10,31,32]$ and reciprocal couplings, respectively. The relative RDDI strength can be quantified by $\xi \equiv k_{s} r_{\mu+1, \mu}$, where $\xi=0$ or $2 \pi$ represents the strongcoupling regime with vanishing dispersions that cannot be achieved in free space [17]. In Fig. 1, we numerically obtain the steady-state phase diagram of a weakly driven chiralcoupled atomic chain at $\theta_{s}=\pi / 2$ and $\delta_{\mu}=0$, where some corresponding normalized excitation distributions $\left(\tilde{P}_{j}\right)$ are shown in Fig. 2. We define normalized excitation populations as

$$
\tilde{P}_{j}=\frac{\sigma_{j}^{(s) \dagger} \sigma_{j}^{(s)}}{\sum_{j=1}^{N} \sigma_{j}^{(s) \dagger} \sigma_{j}^{(s)}} .
$$

The steady-state phases of ETD [see, for example, in Fig. 2(a)] and $\mathrm{CO}$ both show extended features of excitation distributions, which we can characterize by the participation ratio (PR) [33],

$$
\mathrm{PR} \equiv \frac{\left(\sum_{j=1}^{N} \Delta \tilde{P}_{j}\right)^{2}}{\sum_{j=1}^{N}\left(\Delta \tilde{P}_{j}\right)^{2}},
$$

where $\Delta \tilde{P}_{j}=\left|\tilde{P}_{j}-N^{-1}\right| \Theta\left(\tilde{P}_{j}-N^{-1}\right)$ with the Heaviside step function $\Theta$ evaluates the state variations from an ETD phase (narrow region close to $\xi=0$ ) of uniform distributions $N^{-1}$. We further distinguish them by an additional finite structure factor $[S(k)]$ present in the CO phases [see also Fig. 2(b) with alternating excitation patterns on top of the relative flat background populations]. We define the finite structure factor as

$$
S(k)=\sum_{j, m}^{N} e^{i k(j-m)} \tilde{P}_{j} \tilde{P}_{m} .
$$

In Figs. 2(c) and 2(d)-2(f), BEE and BHE phases involve edge and hole excitations at both edges of the chain, and finally we show a region of CFD in Figs. 2(g) and 2(h), where two distinct excitation distributions for even and odd numbers of
TABLE I. Criteria to classify the phases in Fig. 1.

\begin{tabular}{lccc}
\hline \hline Phases & PR & $S(k \neq 0)$ & Edge or hole excitations \\
\hline ETD & PR $\gg 2$ & 0 & No \\
CO & PR $\gg 2$ & Finite & No \\
BEE & PR $=2$ & 0 & Yes \\
BHE & PR $\gg 2$ & 0 & Yes \\
\hline \hline
\end{tabular}

atoms emerge, respectively. In Table I, we show the essential criteria for various phases, and below we discuss in more detail how we classify these phases and regions.

The excitation angle $\theta_{s}$ and field detunings $\delta_{\mu}$ provide extra degrees of freedom for steady states, which would hugely modify the phase diagram presented in Fig. 1. We will not investigate their effects in detail, but will present the essential outcome in Sec. IV, particularly on excitation transport. The role of $\theta_{s}$ manifests in a linearly increasing phase imprinting on the atomic chain, which can lead to multipeaks or relocation of the finite structure factors in the $\mathrm{CO}$ phase. In the BEE and BHE phases, $\theta_{s}$ modifies the portions between bi-edge or bi-hole excitations, which even allows edge-hole excitations at the ends of the chain. The effect of $\delta_{\mu}$ shows mainly in modifying the amount of atomic excitations individually, and therefore we set them under resonant conditions in the phase diagram of Fig. 1 to clearly demonstrate the competition between the directionality of light and the interatomic distances.

\section{A. CO phase}

The states with $\mathrm{CO}$ are determined by finite structure factors $S(k)$. Finite $S(k)$ indicates an emerging structure that behaves as a solid with $\mathrm{CO}$ or an insulator in optical lattices. The CO phase boundary extends symmetrically from $\xi=\pi / 2$ toward two critical points at the lower corners of the diagram in Fig. 1, which also coincides with abrupt changes in the participation ratio PR. When PR is as small as 2 in the BEE phase at $D=0$ in the lower plot of Fig. 1(b), we attribute it to a localized state where most of the atomic excitations occupy both ends of the chain. For PR $\gg 2$, we characterize them as extended or delocalized states.

The phase boundaries of CO-BEE and CO-BHE are determined respectively by abrupt occurrences of edge or hole excitations at both ends of the chain. This coincides with clear differences in the participation ratios between extended and localized states, and further with a distinction from a finite CO. The ETD-CO phase boundary, however, is not obvious since the ETD and CO phases are both extended. Close to this phase boundary, the structure factors are decreasing as $N$ increases. Therefore, we fit the maximum (Max.) of $S(k \neq 0)$ in terms of $N$ by $\left(a N^{-1}+b\right)$ with fitting parameters $a$ and $b$, where a finite $b>0$ represents a finite $S(k)$ in the thermodynamic limit of $N \rightarrow \infty$, and we characterize it as a $\mathrm{CO}$ phase.

We delineate the ETD-CO phase boundary by fitting four points of $N=100-400$ in the phase diagram. As an example, we show the case at $D=0.05$ and extract the maximum of the structure factors at $k \neq 0$, 


\begin{tabular}{lcc}
\hline \hline$N$ & Max. of $S(k)$ at $\xi=0.001$ & Max. of $S(k)$ at $\xi=0.002$ \\
\hline 100 & $4.4 \times 10^{-4}$ & $3.58 \times 10^{-4}$ \\
200 & $9 \times 10^{-5}$ & $6.26 \times 10^{-4}$ \\
300 & $1.09 \times 10^{-4}$ & $3.74 \times 10^{-4}$ \\
400 & $1.56 \times 10^{-4}$ & $3.42 \times 10^{-4}$ \\
\hline \hline
\end{tabular}

where the fitting parameters of $(a, b)=\left(0.043,-3 \times 10^{-5}\right)$ and $\left(-0.0013,4 \times 10^{-4}\right)$, respectively, making $\xi=0.002 \mathrm{a}$ point on the phase boundary separating the ETD and CO phases. Since this boundary is very close to the line of $\xi=0$, we specify the boundary with a precision up to $\xi= \pm 0.001$. From the above table, we note the oscillating $S(k)$ as $N$ increases, and thus more points for fitting make no significant changes in this estimate of the phase boundary.

\section{B. BEE and BHE phases}

As shown in Fig. 1(b), sharp rises in PR emerge when $\xi$ crosses the extended CO or BHE $\left(\tilde{P}_{1(N)}<N^{-1}\right)$ phases. This is in huge contrast to the localized BEE phase $\left(\tilde{P}_{1(N)}>\right.$ $N^{-1}$ ), where PR remains constantly small as the system size increases. At $D=0$, the phase boundary between BEE and BHE starts from $\xi=\pi / 3$ and collapses to $\pi / 2$ as $D$ increases. For an increasing $D$, the PR of the BHE phase is suppressed, and we further distinguish it by different scalings of the system sizes. The strong (s) BHE region suggests significant extended state distributions with hole excitations at both ends of the chain, which endows the emergence of persistent subharmonic dynamics we will present below.

Here, we first show the analytical results for the phase boundary at $\xi=\pi / 3$ from BEE to BHE at $D=0$, and later on below the numerically obtained boundaries between sBHE, mBHE, and wBHE. For $\delta_{\mu}=0$ and $\theta_{s}=\pi / 2$ considered in the phase diagram of Fig. 1, we have the coupling matrix at $D=0$,

$$
M=-\left[\begin{array}{ccccc}
\frac{1}{2} & \frac{1}{2} e^{i \xi} & \frac{1}{2} e^{i 2 \xi} & \cdots & \frac{1}{2} e^{i(N-1) \xi} \\
\frac{1}{2} e^{i \xi} & \frac{1}{2} & \frac{1}{2} e^{i \xi} & \cdots & \frac{1}{2} e^{i(N-2) \xi} \\
\frac{1}{2} e^{i 2 \xi} & \frac{1}{2} e^{i \xi} & \frac{1}{2} & \cdots & \vdots \\
\vdots & \vdots & \ldots & \ddots & \vdots \\
\frac{1}{2} e^{i(N-1) \xi} & \frac{1}{2} e^{i(N-2) \xi} & \cdots & \cdots & \frac{1}{2}
\end{array}\right] \text {, }
$$

which is symmetric, and so is its inverse $M^{-1}$. At the BEEBHE phase boundary, $\tilde{P}_{1(N)}$ should be equal to $N^{-1}$ to distinguish from the edge $\left(\tilde{P}_{1(N)}>N^{-1}\right)$ and hole $\left(\tilde{P}_{1(N)}<N^{-1}\right)$ excitations.

We obtain $\tilde{P}_{j}$ from $M^{-1}$, and take $N=3$ as an example. We then obtain $\tilde{P}_{1}\left(\tilde{P}_{N}=\tilde{P}_{1}\right)$,

$$
\tilde{P}_{1}=\frac{A}{2 A+A^{2}}, \quad A \equiv\left|1-e^{i \xi}\right|^{2},
$$

which gives $\xi=\pi / 3$ from $A=1$ when $\tilde{P}_{1}=1 / 3$. Another solution of $\xi \approx 0$ from $A=0$ is ignored due to a divergent time of $\tilde{P}_{1}$ to reach steady states. This relates to critical regimes, which we will discuss in Sec. III C. For a general
$N$, we obtain the solution of $\xi$ from

$$
\tilde{P}_{1}=\frac{A}{2 A+(N-2) A^{2}}=\frac{1}{N},
$$

which gives again $\xi=\pi / 3$ for $A=1$. In a critical regime when $\xi \approx 0$, we obtain $\tilde{P}_{1(N)}=1 / 2$, which indicates that the atomic excitations are populated equally to the edges of the chain. When $\xi=\pi$, as another critical regime, we have $\tilde{P}_{1(N)}=1 /(4 N)$ for $N \gg 1$, a signature of hole or null excitations at the edges. We investigate the critical regimes in more detail below.

\section{Phase boundaries between $\mathrm{SBHE}, \mathrm{mBHE}$, and wBHE}

In the BHE phase, we note the suppression of PR as $D$ increases, which indicates that BHE becomes less delocalized. To quantify various regions of PR, we again fit the maximum of PR in terms of $N$ by $\beta N^{\alpha}$ with fitting parameters $\beta$ and $\alpha$, where $\alpha$ denotes the degree of scalings for strong (s), moderate $(\mathrm{m})$, and weak (w) regimes with $\alpha>0.5$ (below $D \approx 0.37$ ), $0.1<\alpha<0.5$, and $\alpha<0.1$ (above $D \approx 0.67$ ), respectively. This algebraic dependence of $N$ is suggested at $D=0$, where PR $\propto N$.

Below, we show the PR and their fitting parameters of $\alpha$ around $\mathrm{sBHE}, \mathrm{mBHE}$, and $\mathrm{wBHE}$ regimes by three data points,

\begin{tabular}{lcccc}
\hline \hline$N$ & $\mathrm{PR}(D=0.37)$ & $\mathrm{PR}(0.38)$ & $\mathrm{PR}(0.67)$ & $\mathrm{PR}(0.68)$ \\
\hline 25 & 13.61 & 13.15 & 3.47 & 3.32 \\
50 & 20.89 & 19.87 & 3.82 & 3.61 \\
100 & 27.65 & 25.92 & 4.012 & 3.77 \\
$\alpha$ & 0.51 & 0.49 & 0.105 & 0.092 \\
\hline \hline
\end{tabular}

which sets the boundaries between strong, moderate, and weak BHE phases.

\section{Critical points and a region of CFD}

We further locate two critical points at $D=0, \xi=0$, and $\pi$, respectively, and a phase of CFD which shows very different PRs for an even or odd number of atoms. The critically slow behavior can be identified by the time it takes for the atomic excitations to reach the other end, which has an algebraic dependence of directionality $D$ as shown in Fig. 3(a). This slow equilibrium of the system dynamics also manifests in other extended phases. Criticality arises due to $(N-1)$ decoherence-free modes in the eigenvalues of $(-N / 2,0, \ldots, 0) \gamma$, obtained from $M$, where zero decay modes are not allowed at other $\xi$ 's. These decoherence-free modes mark a distinct region separating them from other noncritical ones at $D=0$ with subradiant sectors of small but finite decay rates. We note that such subradiant modes have been recently investigated on revealing the fermionization feature [34-36] and slow dynamics [35] in an atom-waveguide system.

We demonstrate two more examples of $\mathrm{CO}$ and BEE phases in Fig. 3(b), where a period of eight sites and significant excitations at both edges emerge, respectively. A region of CFD can be characterized by a dichotomy of steady states for an even and odd number of atoms in Fig. 3(c). For the 

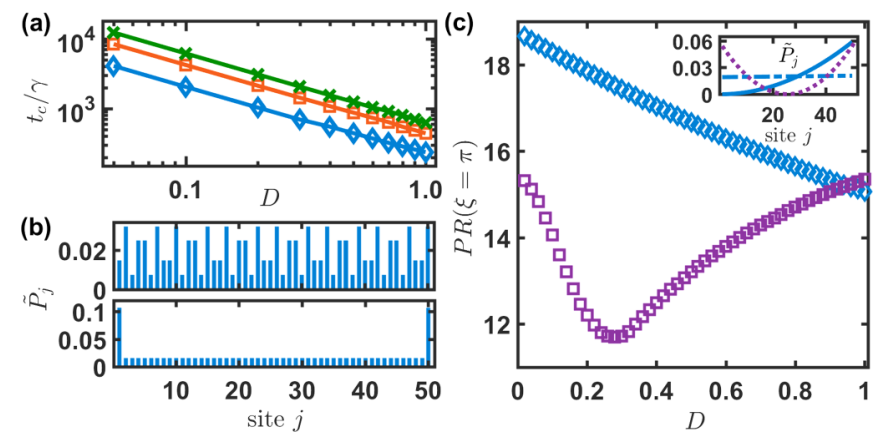

FIG. 3. Characteristics of critical points, $\mathrm{CO}, \mathrm{BEE}$, and a region of CFD. (a) The time $t_{c}$ of atomic excitations in a log-log plot to go through the whole chain and reach the other end for various system sizes $N=50(\diamond), 100(\square)$, and $150(\times)$, at $\xi=0$. (b) A demonstration of states of $\mathrm{CO}(D=1, \xi=\pi / 4)$ and $\operatorname{BEE}(D=0$, $\xi=\pi / 8)$ in the upper and lower panels, respectively, for $N=50$. (c) CFD region shows two different PR for $N=50(\diamond)$ and 51 ( $\square$ ), where state distributions $\tilde{P}_{j}$ in the inset present the cases of $D=1$ (solid) and 0.02 (dashed dotted) for $N=50$, and $D=0.02$ for $N=51$ (dots).

first impression with a finite $D$, we expect a flow of atomic excitations toward the preferred direction with its minimum in the opposite side. By contrast, another configuration with its minimum moving toward the center of the chain as $D$ decreases shows up in an odd number of atoms. It is the extra atom in an odd chain that distinguishes the two configurations near $D=0$ as shown in the inset of Fig. 3(c), which should satisfy the inversion symmetry as $D \rightarrow 0$. An even (odd) number of atoms in this particular region presents a balanced (unbalanced) RDDI of alternating $\pm \gamma_{R / L}$, and this dichotomy can be further classified by two distinguishing PRs. The more delocalized state for an even chain reaches its maximal PR close to the critical point, whereas the PR for an odd case never exceeds the value at $D=1$, and instead shows a minimum at a finite and universal value of $D=0.28$. We note that the cross of PR near $D=1$ is due to the finite-size effect, which collapses in the thermodynamic limit.

\section{TRANSPORT OF ATOMIC EXCITATIONS AND EMERGENCE OF CRYSTALLINE ORDER}

Next, we investigate the effect of spatially dependent $\delta_{\mu}$ and $\theta_{s}$, which leads to the redistribution of atomic excitations. We quantify the transport of atomic excitations by their difference between the left and right parts of the chain [37],

$$
T_{p}=\frac{\sum_{\mu=1}^{(N-1) / 2} \tilde{P}_{\mu}-\sum_{\mu=(N+3) / 2}^{N} \tilde{P}_{\mu}}{\sum_{\mu=1}^{N} \tilde{P}_{\mu}},
$$

where we have excluded the central one for an odd $N$. Positive or negative $T_{p}$ represents that the left or right parts of the chain are more occupied, and right (left) linearly increasing $\delta_{\mu}$ should favor positive (negative) $T_{p}$ since atoms are less excited under off-resonant driving fields, in the sense of a noninteracting regime with negligible RDDI. By contrast in Fig. 4(a), for an onset of a small slope of linearly increasing detunings to the right, the interaction-driven transport changes a positive $T_{p}$ to a negative one when $\xi \gtrsim \pi / 8$, comparing $T_{p}=$ 0 for both BEE and sBHE phases at $D=0$ with a vanishing slope (symmetric distributions with inversion symmetry). This is more evident for moderate slopes, where negative $T_{p}$ shows up in different ranges, presenting the competition between the relative RDDI strength and excitation detunings, that is, the
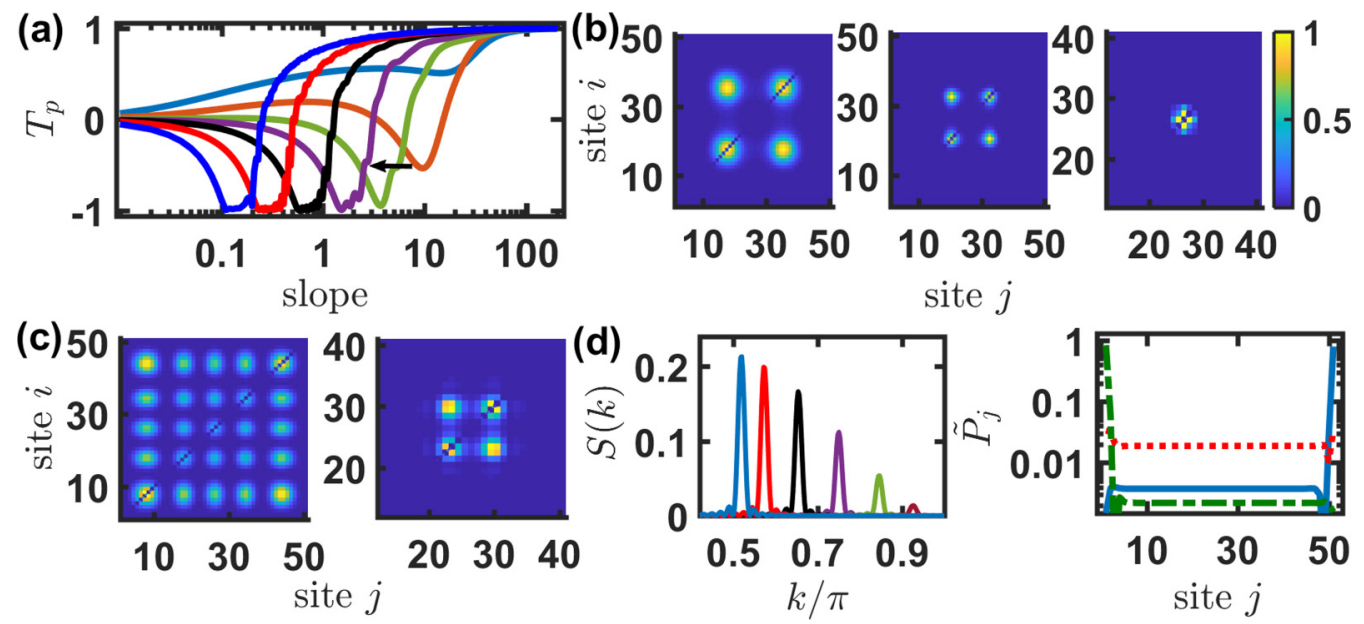

FIG. 4. Transport and localization of atomic excitations, emergence of $\mathrm{CO}$, and the effect of $\theta_{s}$. (a) Interaction-driven transport of atomic excitations vs slopes (s) of linearly increasing $\delta \mu=(s / N)(\mu-1)$. Arrows indicate the cases of $\xi=\pi / 32, \pi / 16, \pi / 8, \pi / 4, \pi / 2,3 \pi / 4$, and $7 \pi / 8$, at $D=0$. (b) A localization of atomic excitations appears in atom-atom correlations $\tilde{P}_{i} \tilde{P}_{j}$ under a harmonic-potential-like $\delta_{\mu}=$ $h[\mu-(N+1) / 2]^{2}$ with $h=N^{-1}$ as $\xi$ increases from $\pi / 4$ (left), $\pi / 2$ (middle), to $3.9 \pi / 4$ (right), at $D=0$. (c) Edge accumulation is evident in $\tilde{P}_{i} \tilde{P}_{j}$ at $D=1$ with $\xi=3 \pi / 4$ in the left, and in the right, broad COs emerge at $D=0.01$ and $\xi=\pi$, both under a smaller $h=0.01 N^{-1}$ of harmonic-potential-like detunings with the same color bar in (b). (d) Finite structure factors $S(k)$ move from low to high $k$ from $\theta_{s}=$ $7 \pi / 8,6 \pi / 8, \ldots$, to $2 \pi / 8$, at $D=1, \xi=\pi / 4$, and $N=101$. In the right panel, for $D=0.3, \xi=\pi / 4$, the localized edge excitation moves from the right end at $\theta_{s}=7 \pi / 8$ (solid), to the left end at $\theta_{s}=\pi / 8$ (dashed dotted), comparing the BEE phase at $\theta_{s}=\pi / 2$ (dots). $N=51$ for (a)-(c) and the right panel of (d). 
case with a larger $\xi$ covers less ranges of slopes for negative $T_{p}$, indicating the lesser effect of RDDI on the transport properties. Eventually all $T_{p}$ turn to a positive side under large slopes as in noninteracting regimes.

Other than linearly increasing detunings, which act as a linear potential to the atomic excitations, here we further focus on a harmonic-potential-like detuning and study how it will modify the transport properties and initiate a localization of atomic excitations. Under this specially designed detuning, the system can simulate particles in a harmonic potential, where a localization or crystalline orders of atomic excitations can emerge by tuning the relative RDDI strengths. In Fig. 4(b) we show the atom-atom correlations which highlight the central localization of excitations as $\xi$ increases under a harmonic-potential-like detuning. This presents the repulsion of atomic excitations in the BEE phase and the dominance of an external potential over the hole excitations in the sBHE phase. A central localization always exists under a tight harmonic detuning, which also indicates a broadened $\mathrm{CO}$. We further show an example of edge accumulations induced from the $\mathrm{CO}$ phase and finite $\mathrm{CO}$ emerging from CFD regions in Fig. 4(c). Edge accumulation in this particular regime is interesting since an additional harmonic detuning allows a flow of excitations toward the edges on top of $\mathrm{CO}$, as if breaking the balanced interaction parameters of $D$ and $\xi$. The emerging $\mathrm{CO}$ in CFD regions reveals again the interference structure between an inward excitation due to an external potential and a repulsion from chiral flow of the atomic excitation. As $D$ increases, chiral flow overtakes the external potential and $\mathrm{CO}$ disappears.

On the other hand, in Fig. 4(d) the effect of excitation angles $\theta_{s}$ manifests in moving the locations of the finite structure factors by imprinting spatially dependent phases on the chain, and in relocating the edge excitations to the left- or rightmost of the chain as if a strong linear potential is induced for single-edge excitations. A joint manipulation of excitation detunings and angles thus enables the controllable transport of localized atomic excitations.

\section{PERSISTENT SUBHARMONIC OSCILLATIONS}

Here, we present the long-time dynamics of equilibration in steady states, specifically in the sBHE phase which shows strong extended features of hole excitations. This nonequilibrium dynamics is particularly interesting since it shows an inability of systems to explore all configurations as in integrable systems with many conserved quantities. Below we demonstrate similar long-time dynamics of atomic excitations in a driven-dissipative chiral-coupled atomic chain, where a signature of oscillations with two timescales and a particular pattern of excitation distributions emerges. This may give insights into a new universality class of quantum dynamics that is interaction driven within a subset of many-body states [38]. There is no definite region for this collectively coupled dynamics in a chiral-coupled atomic chain, but can be approximately confined in $\mathrm{mBHE}$ and $\mathrm{sBHE}$ for $D \lesssim 0.5$ and $\mathrm{BEE}$ for smaller $D$.

In Fig. 5, we show two examples in the noncascaded $(D \neq 0)$ and reciprocal $(D=0)$ coupling regimes. The noncascaded
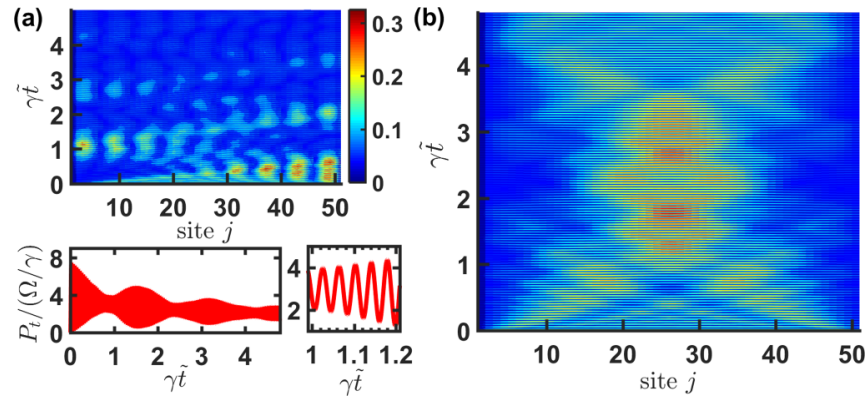

FIG. 5. Subharmonic oscillations and butterflylike dynamics in the sBHE phase. (a) Time evolutions of $\tilde{P}_{j}$ and subharmonic oscillations of total populations $P_{t}=\sum_{\mu=1}^{N} \sigma_{\mu}^{\dagger} \sigma_{\mu}$ (rescaled by $\Omega / \gamma$ ) in the upper and lower panels, respectively, at $D=0.2$ and $\xi=0.8 \pi$. (b) Butterflylike time dynamics of $\tilde{P}_{j}$ at $D=0$ and $\xi=0.8 \pi$ with the same color bar in (a). A rescaled time $\tilde{t}=1000 t$ represents the long-time dynamics before reaching an equilibrium.

time dynamics shows an oscillating spread of populations from left to right initially since $\gamma_{R}>\gamma_{L}$, and then back and forth until equilibrium is reached, similar to quantum Newton's cradle [39] that breaks ergodicity. Furthermore, in the lower panel of Fig. 5(a), we find an oscillation of total population $P_{t}$ with two timescales, a signature of persistent subharmonic evolution which is related to temporal correlations and discrete time-crystalline orders [40] in many dipolar spin impurities in diamond. The slow thermalization in Fig. 5(a) and its timescale is purely interaction driven in sBHE phases, which can be further enhanced in the reciprocal coupling regime with much longer time responses.

In Fig. 5(b), we show the reoccurring patterns resembling a butterfly under reciprocal couplings. The agglomeration of atomic excitations resides around the center of the chain before expanding to the edges, which we attribute again as nonergodic and is associated with nonequilibrium many-body scars [38]. The so-called scars relate to some special eigenstates that have high probabilities of a subset of a system's Hilbert space. The concept of scars is originally discussed in isolated and unstable periodic orbits allowed in classical chaotic systems [41], where a concentration of eigenfunctions forms and the ergodicity of the system is broken. Similarly in the driven-dissipative chiral-coupled systems we consider here, eventually a subset of subradiant eigenstates is populated as time evolves, which can further lead to some structure or scars in the system dynamics.

To have some clues on the relation between subradiant sectors and long-time dynamics in Fig. 5, we obtain the eigenspectrum directly from the coupling matrix $M$ along $D=0$. We find the emergence of significant energy splittings appearing toward the lowest subradiant sectors as $\xi$ increases from $\operatorname{BEE}(\xi<\pi / 3)$ to $\operatorname{sBHE}(\xi>\pi / 3)$ phases in Fig. 6. Since the long-time behaviors of subharmonic oscillations observed in Fig. 5 are more significant in sBHE phases, we attribute the nonergodic oscillations to the highly dispersive subradiant sectors of the spectrum.

This can be further clarified in Fig. 7, where we plot the frequency shifts in an ascendant direction. Again, as we go from BEE to sBHE phases, the eigenfrequency shifts close to 


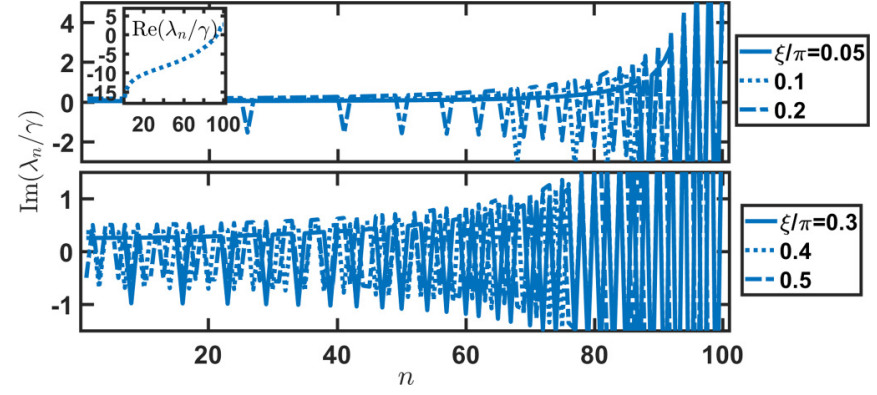

FIG. 6. Frequency shifts of the eigenspectrum for $N=100$. Here, we sort the eigenvalues $\operatorname{Im}\left(\lambda_{n}\right)$ according to ascendant decay rates of $\operatorname{Re}\left(\lambda_{n}\right)$. In the upper panel, the inset shows the case of $\xi / \pi=$ 0.1 in logarithmic scales, and note that the trend of the logarithmic scales of eigen decay rates is similar for other cases of $\xi$, except some larger superradiant or lower subradiant eigenvalues appearing for different $\xi$ 's. We specifically focus on the subradiant sectors of $n \lesssim 80$, which is way below $\operatorname{Re}\left(-\lambda_{n}\right)=\gamma$.

$\operatorname{Im}\left(\lambda_{n}\right)=0$ start to split, and an energy-gap-like jump appears from the red-detuned to blue-detuned shifts. This opening of an energy gap corresponds to the subradiant sectors which allow relatively lower decay rates with finite energy shifts. From the perspective of steady states under driven-dissipative settings, these constrained subradiant states are responsible for the persistent subharmonic oscillations or butterflylike time dynamics that are far from equilibrium. We note that the understanding of nonergodic dynamics in quantum systems is still an ongoing research, and there are questions such as how ergodicity can be strongly or weakly broken [38], and what are the essential factors of a model that manifests long-time behaviors. At the least, the results shown here (i) specify that a unique driven-dissipative chiral-coupled system with RDDI can also establish nonequilibrium dynamics, (ii) relate subradiant sectors with an onset of energy splittings to nonergodic oscillations, and (iii) suggest that the chirality of the system with unidirectional couplings or larger directionality $D$ in our studies hinders the long-time dynamics. As a final remark, we denote the scale of $P_{t}$ in Fig. 5 by $\Omega / \gamma$ instead of $(\Omega / \gamma)^{2}$, showing the excitation enhancement due to the collective couplings of RDDI, compared to the noninteracting case which should be an order of $(\Omega / \gamma)^{2}$.

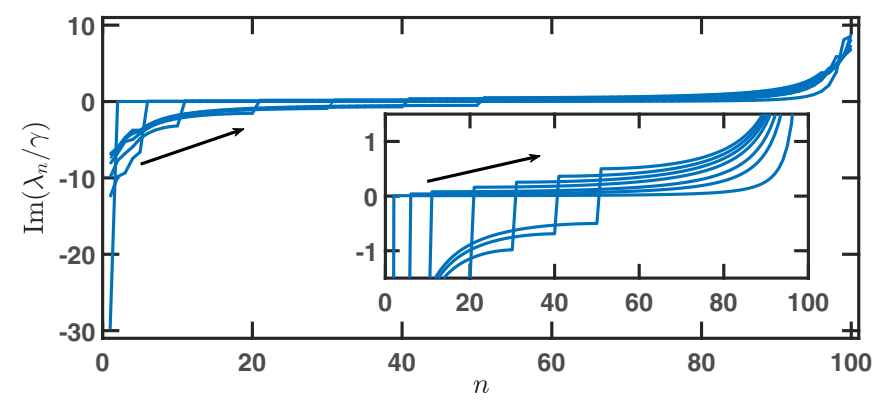

FIG. 7. Frequency shifts of the eigenspectrum for $N=100$ from low to high values as $n$ increases. The inset zooms in the values near $\operatorname{Im}\left(\lambda_{n}\right)=0$, and the arrows marks the direction of increasing $\xi / \pi=0.01,0.05,0.1-0.5$.

\section{CONCLUSION}

In conclusion, the chiral-coupled atomic chain presents fruitful interaction-driven quantum phases of matter under driven-dissipative settings, with competing interactions between relative strengths of the 1D RDDI, directionality of decay channels, and external excitation conditions. Under a weak excitation field, the system shows critically slow behaviors, crystalline orders, localized edge or extended hole excitations, and a region of states dichotomy for an even or odd number of quantum emitters. The nonequilibrating states of extended hole excitations specifically manifest longtime oscillations, in huge contrast to the states with $\mathrm{CO}$ and phases of BEE and wBHE close to the unidirectional coupling regime. Future directions can lead to unraveling a clear mechanism for the initiation of persistent subharmonic time evolutions, its relation to ergodicity of the chiral-coupled system, or many-body simulations of exotic or topological states in nonreciprocal quantum optical systems.

\section{ACKNOWLEDGMENTS}

We acknowledge the support from the Ministry of Science and Technology (MOST), Taiwan, under Grant No. MOST106-2112-M-001-005-MY3 and thank Y.-C. Chen, G.-D. Lin, and M.-S. Chang for insightful discussions.

\section{APPENDIX: HIERARCHY-COUPLED EQUATIONS}

From a general model of a driven-dissipative chiralcoupled atomic chain in Eq. (1), we are able to derive the coupled equations with a hierarchy of multiple atomic correlations. We will see below that the hierarchy arises due to the resonant dipole-dipole interactions of $H_{L(R)}$ and $\mathcal{L}_{L(R)}[\rho]$.

As a demonstration of hierarchy-coupled equations, we consider a uniform side excitation field with a Rabi frequency $\Omega$ at a right angle $\theta_{s}=\pi / 2$. First, the time evolution of the coherence operators $\sigma_{\mu}$ reads

$$
\begin{aligned}
\dot{\sigma}_{\mu}= & \left(i \delta_{\mu}-\frac{\gamma_{L}+\gamma_{R}}{2}\right) \sigma_{\mu}+i \Omega\left(\sigma_{\mu}^{e e}-\sigma_{\mu}^{g g}\right) \\
& -\gamma_{L} \sum_{\nu>\mu} e^{i k_{s}\left|r_{\mu, \nu}\right|}\left(\sigma_{\mu}^{g g}-\sigma_{\mu}^{e e}\right) \sigma_{\nu} \\
& -\gamma_{R} \sum_{\nu<\mu} e^{i k_{s}\left|r_{\mu, \nu}\right|}\left(\sigma_{\mu}^{g g}-\sigma_{\mu}^{e e}\right) \sigma_{\nu},
\end{aligned}
$$

where $\gamma_{L(R)}$ respectively quantifies the couplings between the atom to the rest of left (right) of the chain. Next, the evolution of the excitation population reads

$$
\begin{aligned}
\dot{\sigma}_{\mu}^{e e}= & i \Omega \sigma_{\mu}-i \Omega \sigma_{\mu}^{\dagger}-\gamma_{L} \sum_{\nu>\mu}\left(e^{i k\left|r_{\mu, \nu}\right|} \sigma_{\mu}^{\dagger} \sigma_{\nu}+\text { H.c. }\right) \\
& -\gamma_{R} \sum_{\nu<\mu}\left(e^{i k\left|r_{\mu, \nu}\right|} \sigma_{\mu}^{\dagger} \sigma_{\nu}+\text { H.c. }\right),
\end{aligned}
$$

where H.c. is the Hermitian conjugate. We can see that the above two single atomic operators couple with each other via two-body correlations of $\sigma_{\mu}^{g g} \sigma_{v}, \sigma_{\mu}^{e e} \sigma_{\nu}$, and $\sigma_{\mu}^{\dagger} \sigma_{\nu}$. This indicates the hierarchy relations between $n$th and $(n+1)$ th moments of operators up to $N$ th ones. 
To have another taste of a hierarchy-coupled equation of two-body correlations, we show the time evolution of $\sigma_{\mu}^{\dagger} \sigma_{v}$ as an example, which reads

$$
\begin{aligned}
\frac{d\left(\sigma_{\mu}^{\dagger} \sigma_{v}\right)}{d t}= & -\left(\gamma_{R}+\gamma_{L}\right) \sigma_{\mu}^{\dagger} \sigma_{v}-i \Omega\left[\left(\sigma_{\mu}^{e e}-\sigma_{\mu}^{g g}\right) \sigma_{v}-\sigma_{\mu}^{\dagger}\left(\sigma_{v}^{e e}-\sigma_{v}^{g g}\right)\right] \\
& -\gamma_{L}\left[\sum_{\alpha>v} e^{i k\left|r_{\alpha, v}\right|} \sigma_{\mu}^{\dagger}\left(\sigma_{v}^{g g}-\sigma_{v}^{e e}\right) \sigma_{\alpha}+\sum_{\alpha>\mu} e^{-i k\left|r_{\alpha, \mu}\right|}\left(\sigma_{\mu}^{g g}-\sigma_{\mu}^{e e}\right) \sigma_{\alpha}^{\dagger} \sigma_{v}\right] \\
& -\gamma_{R}\left[\sum_{\alpha<v} e^{i k\left|r_{\alpha, v}\right|} \sigma_{\mu}^{\dagger}\left(\sigma_{v}^{g g}-\sigma_{v}^{e e}\right) \sigma_{\alpha}+\sum_{\alpha<\mu} e^{-i k\left|r_{\alpha, \mu}\right|}\left(\sigma_{\mu}^{g g}-\sigma_{\mu}^{e e}\right) \sigma_{\alpha}^{\dagger} \sigma_{v}\right],
\end{aligned}
$$

where three-body operators emerge. In treating the effect of quantum fluctuations in dissipative quantum optical systems [29], we can usually truncate the hierarchy up to two-body noise correlations due to the stochastic nature of the quantum noise. For a general driven-dissipative system we consider here, many-body states can be all explored (a total of $2^{N}$ configurations for $N$ two-level quantum registers), and this is exactly the merit of the quantum resource for quantum information processing and quantum computation. However, this also prevents appropriate numerical simulations of the general quantum dynamics by classical computers, except for systems with smaller higher-order moments or with a technique of matrix product states which are efficient in finding the many-body ground states.

Next, we simplify the hierarchy-coupled equations by taking a low saturation regime, where $\sigma_{\mu}^{g g} \approx 1 \gg \sigma_{\mu}^{e e}$. Under this condition, Eq. (A1) reduces to

$$
\begin{aligned}
\dot{\sigma}_{\mu}= & \left(i \delta_{\mu}-\frac{\gamma_{L}+\gamma_{R}}{2}\right) \sigma_{\mu}-i \Omega-\gamma_{L} \sum_{\nu>\mu} e^{i k_{s}\left|r_{\mu, \nu}\right|} \sigma_{\nu} \\
& -\gamma_{R} \sum_{\nu<\mu} e^{i k_{s}\left|r_{\mu, \nu}\right|} \sigma_{\nu},
\end{aligned}
$$

and the evolutions of all other higher-order moments can be decomposed in terms of the above one. For example, $\left\langle\sigma_{\mu}^{\dagger} \sigma_{\nu}\right\rangle=\left\langle\sigma_{\mu}^{\dagger}\right\rangle\left\langle\sigma_{\nu}\right\rangle$, where $\langle\cdot\rangle$ denotes the expectation values, such that $d\left\langle\sigma_{\mu}^{\dagger} \sigma_{\nu}\right\rangle / d t=\left\langle\dot{\sigma}_{\mu}^{\dagger}\right\rangle\left\langle\sigma_{\nu}\right\rangle+\left\langle\sigma_{\mu}^{\dagger}\right\rangle\left\langle\dot{\sigma}_{\nu}\right\rangle$. In the main text, we solve the steady-state solutions of Eq. (A4), which manifest many interesting quantum phases in the steady state.
[1] P. Lodahl, S. Mahmoodian, S. Stobbe, A. Rauschenbeutel, P. Schneeweiss, J. Volz, H. Pichler, and P. Zoller, Nature (London) 541, 473 (2017).

[2] D. E. Chang, J. S. Douglas, A. González-Tudela, C.-L. Hung, and H. J. Kimble, Rev. Mod. Phys. 90, 031002 (2018).

[3] R. Mitsch, C. Sayrin, B. Albrecht, P. Schneeweiss, and A. Rauschenbeutel, Nat. Commun. 5, 5713 (2014).

[4] I. J. Luxmoore, N. A. Wasley, A. J. Ramsay, A. C. T. Thijssen, R. Oulton, M. Hugues, S. Kasture, V. G. Achanta, A. M. Fox, and M. S. Skolnick, Phys. Rev. Lett. 110, 037402 (2013).

[5] I. Söllner et al., Nat. Nanotechnol. 10, 775 (2015).

[6] M. Arcari, I. Söllner, A. Javadi, S. Lindskov Hansen, S. Mahmoodian, J. Liu, H. Thyrrestrup, E. H. Lee, J. D. Song, S. Stobbe, and P. Lodahl, Phys. Rev. Lett. 113, 093603 (2014).

[7] K. Y. Bliokh, A. Y. Bekshaev, and F. Nori, Nat. Commun. 5, 3300 (2014).

[8] K. Y. Bliokh and F. Nori, Phys. Rep. 592, 1 (2015).

[9] A. González-Tudela and D. Porras, Phys. Rev. Lett. 110, 080502 (2013).

[10] K. Stannigel, P. Rabl, and P. Zoller, New J. Phys. 14, 063014 (2012).

[11] T. Ramos, H. Pichler, A. J. Daley, and P. Zoller, Phys. Rev. Lett. 113, 237203 (2014).

[12] H. Pichler, T. Ramos, A. J. Daley, and P. Zoller, Phys. Rev. A 91, 042116 (2015).

[13] C. L. Hung, A. Gonzáles-Tudela, J. I. Cirac, and H. J. Kimble, Proc. Natl. Acad. Sci. USA 113, E4946 (2016).
[14] J. Kumlin, S. Hofferberth, and H. P. Büchler, Phys. Rev. Lett. 121, 013601 (2018).

[15] S. Mahmoodian, M. Čepulkovskis, S. Das, P. Lodahl, K. Hammerer, and A. S. Sørensen, Phys. Rev. Lett. 121, 143601 (2018).

[16] P. Solano, P. Barberis-Blostein, F. K. Fatemi, L. A. Orozco, and S. L. Rolston, Nat. Commun. 8, 1857 (2017).

[17] R. H. Lehmberg, Phys. Rev. A 2, 883 (1970).

[18] S. Diehl, A. Micheli, A. Kantian, B. Kraus, H. P. Büchler, and P. Zoller, Nat. Phys. 4, 878 (2008).

[19] B. Kraus, H. P. Büchler, S. Diehl, A. Kantian, A. Micheli, and P. Zoller, Phys. Rev. A 78, 042307 (2008).

[20] F. Verstraete, M. M. Wolf, and J. I. Cirac, Nat. Phys. 5, 633 (2009).

[21] K. Baumann, C. Guerlin, F. Brennecke, and T. Esslinger, Nature (London) 464, 1301 (2010).

[22] H. Weimer, M. Müller, I. Lesanovsky, P. Zoller, and H. P. Büchler, Nat. Phys. 6, 382 (2010).

[23] J. T. Barreiro, M. Müller, P. Schindler, D. Nigg, T. Monz, M. Chwalla, M. Hennrich, C. F. Roos, P. Zoller, and R. Blatt, Nature (London) 470, 486 (2011).

[24] S. Diehl, E. Rico, M. A. Baranov, and P. Zoller, Nat. Phys. 7, 971 (2011).

[25] C.-E. Bardyn, M. A. Baranov, C. V. Kraus, E. Rico, A. İmamoğlu, P. Zoller, and S. Diehl, New J. Phys. 15, 085001 (2013). 
[26] M. Höning, M. Moos, and M. Fleischhauer, Phys. Rev. A 86, 013606 (2012).

[27] S. Diehl, A. Tomadin, A. Micheli, R. Fazio, and P. Zoller, Phys. Rev. Lett. 105, 015702 (2010).

[28] H. J. Carmichael, Statistical Methods in Quantum Optics 1: Master Equations and Fokker-Planck Equations (Springer, Berlin, 2003).

[29] H. J. Carmichael, Statistical Methods in Quantum Optics 2: Non-Classical Fields (Springer, Berlin, 2008).

[30] A. L. Fetter and J. D. Walecka, Quantum Theory of ManyParticle Systems (Dover, New York, 2003).

[31] C. W. Gardiner, Phys. Rev. Lett. 70, 2269 (1993).

[32] H. J. Carmichael, Phys. Rev. Lett. 70, 2273 (1993).

[33] N. C. Murphy, R. Wortis, and W. A. Atkinson, Phys. Rev. B 83, 184206 (2011).
[34] A. Albrecht, L. Henriet, A. Asenjo-Garcia, P. B Dieterle, O. Painter, and D. E. Chang, New J. Phys. 21, 025003 (2019).

[35] L. Henriet, J. S. Douglas, D. E. Chang, and A. Albrecht, Phys. Rev. A 99, 023802 (2019).

[36] Y.-X. Zhang and K. Mølmer, Phys. Rev. Lett. 122, 203605 (2019).

[37] H. H. Jen, J. Phys. B: At. Mol. Opt. Phys. 52, 065502 (2019).

[38] C. J. Turner, A. A. Michailidis, D. A. Abanin, M. Serbyn, and Z. Papić, Nat. Phys. 14, 745 (2018).

[39] T. Kinoshita, T. Wenger, and D. S. Weiss, Nature (London) 440, 900 (2006).

[40] S. Choi et al., Nature (London) 543, 221 (2017).

[41] E. J. Heller, Phys. Rev. Lett. 53, 1515 (1984). 